\title{
The Path to Enhance Recovery after Colorectal Surgery
}

M. Remelhe, O. Santos, S. Brito, C. Mateus, C. Cavaleiro, H. Machado Centro Hospitalar do Porto

\section{INTRODUCTION}

Enhanced Recovery After Surgery (ERAS) care pathways reduce surgical stress, maintain postoperative physiological function and enhance mobilization after surgery, reducing morbidity and length of hospital stay (LOHS).

The aim of this study was to evaluate the institutional compliance with a perioperative ERAS path and patients' outcome in colorectal surgery (CS).

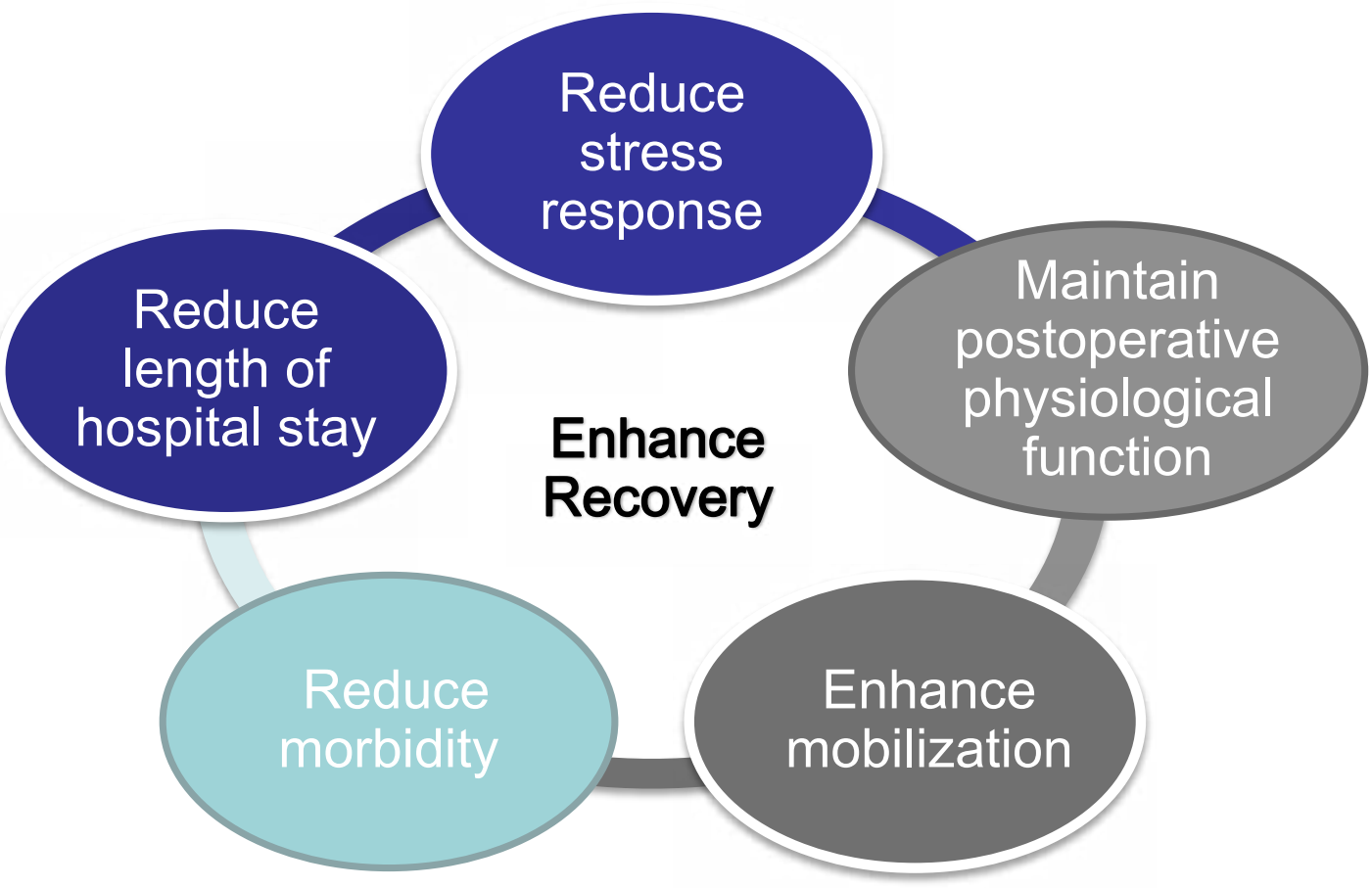

\section{METHOD}

A retrospective audit was conducted after Quality, Health and Safety Department approval. Data were collected from elective CS patients' electronic medical records (2014-2016). Analyzed variables: age, gender, American Society of Anesthesiologists Physical Status (ASA), comorbidities, Colorectal Physiological and Operative Severity Score for the enumeration of Mortality and Morbidity (CR POSSUM), compliance with perioperative ERAS items, LOHS, and perioperative complications, using the ClavienDindo classification (CDc). Descriptive statistical analysis, Whitney and Spearman correlation and t-student test were run in SPSS $\AA^{\circ}$ version24.

\section{RESULTS AND DISCUSSION}

272 patients' medical records were analyzed ( $\bar{x}: 64$ years) (18-89), 145 males, 127 females. ASA 2- 171 (63\%) 3- 82 (30\%). The most common comorbidities were type 2 diabetes and congestive heart failure. CR POSSUM Mortality 3.3\% (SD 4.1)
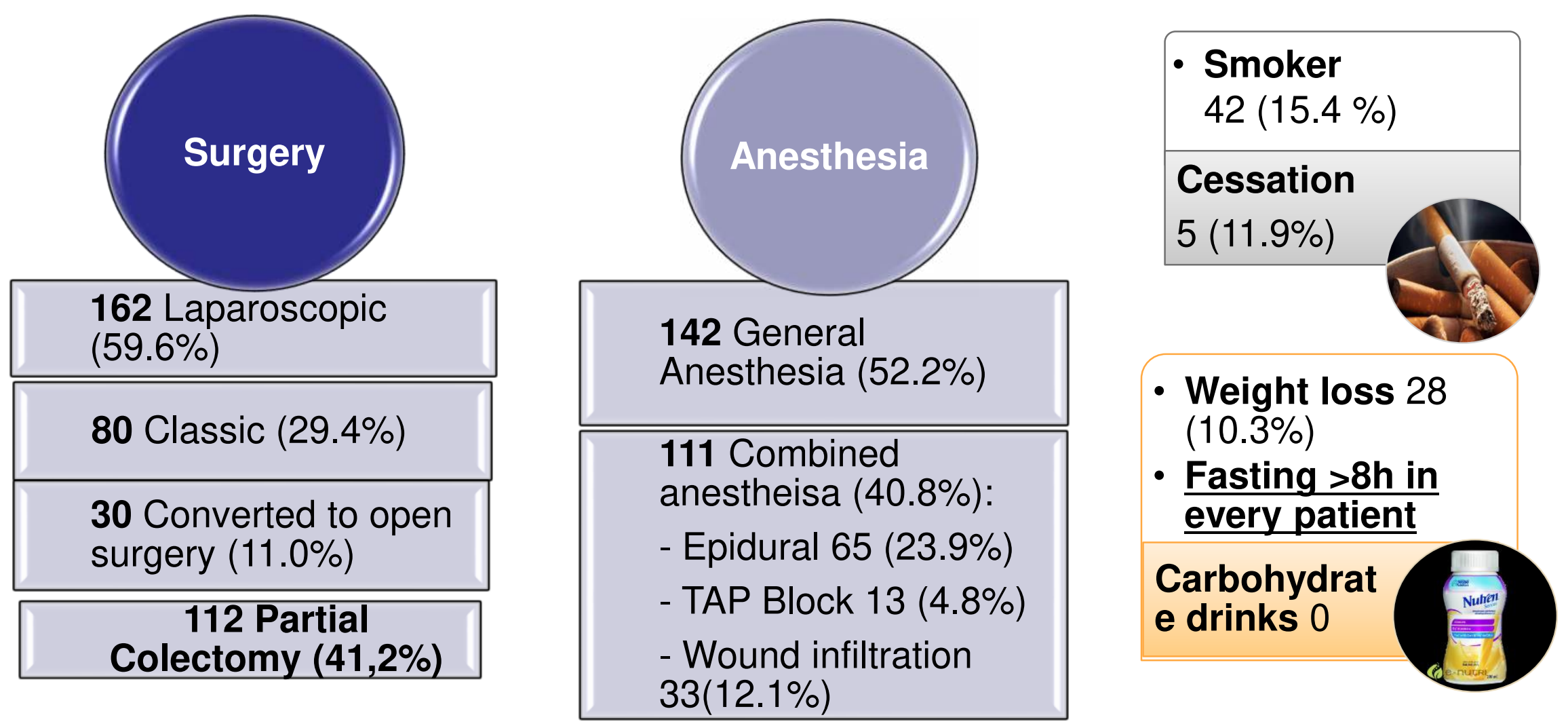

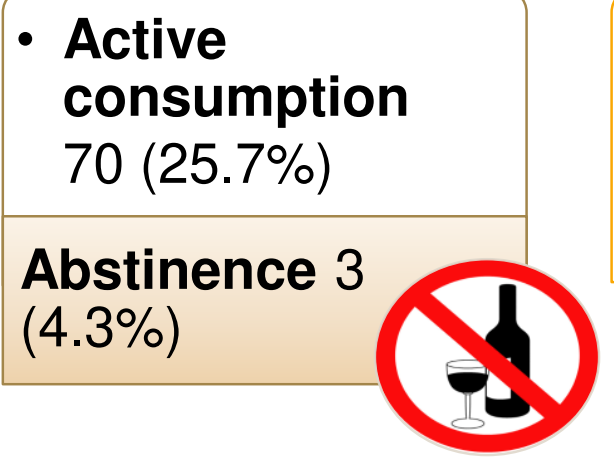

- Anemia 72(26.5\%)

- Blood transfusion intraop. 21 (7.7\%)

Correction $46(63.9 \%)$

- Compression stockings: 233 $(85.7 \%)$

- LMWH 263 (96.7\%)

- Benzodiazepines: $125(46.0 \%)$

- Bowel Preparation 190 (69.9\%)

A positive relation was observed between the first feeding and mobilization day, the beginning of gastrointestinal transit and LOHS $(p<0.05)$.

Difference in mean LOHS laparoscopic $(=9,7 / S D 6.71)$ vs laparotomy or conversion $(=\mathbf{1 7}, \mathbf{9} / \mathrm{SD} 26,81)$ statistically significant $(p<0.05)$

Vs ERAS 6,5d 1

The CDc grade was positively related to CR POSSUM physiological score $(r=0.408 ; p<0.001)$.

There was a statistically significant difference between the means in CR POSSUM physiologic $(U=541.5, p=0.007)$ and mortality scores and a CDc grade $>$ III $(U=525, p=0.004)$.

\begin{tabular}{|cc|}
$\begin{array}{c}\text { Clavien-Dindo } \\
\text { Classification }\end{array}$ & $\mathbf{N}(\%)$ \\
\hline I & $8(2.9 \%)$ \\
\hline II & $28(10.3 \%)$ \\
\hline IIla & $3(1.1 \%)$ \\
\hline IIIb & $6(2.2 \%)$ \\
\hline Iva & $20(7.4 \%)$ \\
\hline Ivb & $16(5.9 \%)$ \\
\hline V & $1(0.4 \%)$ \\
\hline
\end{tabular}

Morbility : 30,1\%.

vs ERAS $12,9 \% \underline{1}$

\section{CONCLUSION}

It is important to audit clinical practice in order to identify factors that may affect the patients' outcome. The path to an ERAS protocol implementation in our institution is a gradual but critical process to improve patients' outcome in CS. 